\title{
Comparative Evaluation of Clinical and Radiographic Outcomes of Indirect Pulp Treatment with Silver Diamine Fluoride versus Calcium Hydroxide in Primary Teeth: A Randomized Controlled Trial
}

\author{
Devendra Patil ${ }^{1}$, Farhin Katge ${ }^{2}$, Khushboo Jain ${ }^{3}$, Manohar Poojari ${ }^{4}$, \\ Vamsi Krishna Chimata ${ }^{5}$ \\ ${ }^{1}$ Senior Lecturer, ${ }^{2}$ Professor and Head, ${ }^{3}$ Post Graduate, ${ }^{4,5}$ Reader, \\ Department of Paediatric and Preventive Dentistry, Terna Dental College, Navi Mumbai, India.
}

Corresponding Author: Farhin Katge

\begin{abstract}
Background: Indirect pulp treatment (IPT) has been a minimally invasive procedure for deep dentinal caries with considerable success. IPT is a procedure which involves excavation of infected dentin and keeping affected dentin intact, followed by application of IPT agent and restoration. The aim of the study was to evaluate and compare clinical and radiographic outcomes of IPT with silver diamine fluoride (SDF) and calcium hydroxide in primary teeth.

Materials and Methods: The present study is a prospective, parallel, randomized controlled trial. The study was conducted with a sample size of 50 primary molars in 4 to 7 year old children. In Group A, 25 teeth each were considered for SDF whereas, 25 teeth for calcium hydroxide IPT (Group B). Clinical and radiographic outcomes were evaluated at baseline, three and six months and were compared for both the groups. Data obtained was analyzed using Fisher's exact test. Level of significance was set at $\mathrm{p}<0.05$.

Results: On statistical analysis of clinical and radiographic outcomes between both the groups IPT using SDF showed 96\% success rate at six month follow up whereas IPT using calcium hydroxide showed $88 \%$ success. However, there was no statistically significant difference found between the groups.
\end{abstract}

Conclusions: SDF can be used as an effective alternative of calcium hydroxide for IPT in primary molars.

Keywords: Indirect pulp treatment, Calcium hydroxide, Silver diamine fluoride, Primary teeth

\section{INTRODUCTION}

Dentistry has now shifted towards minimally invasive procedures. In deep dentinal caries, the superficial infected dentin layer contains majority of microorganisms which requires complete removal. Whereas the affected dentin which is deeper contains minimal amount of microorganisms whichhasremineralization property and can be left intact. [1,2] Indirect pulp treatment (IPT) is one such minimal invasive procedure in which the caries closest to the pulp tissue is left in place and covered with a biocompatible material. The tooth later is restored to prevent microleakage. The aim here is to preserve dental substrate while maintaining pulp vitality, avoiding direct intervention on the pulp tissue. [3]

The agents used for IPT are calcium hydroxide, dentin bonding agents, zinc oxide eugenol, glass ionomer cement and many more. Calcium hydroxide is 
considered as a gold standard for IPT. It has the ability to inhibit bacterial enzymes by means of hydroxyl ions. These ions act on the cytoplasmic membrane of bacteria generating antibacterial effect and activate tissue enzymes such as alkaline phosphatase, which has an influence on mineralization. The mineralized tissue formation on contact with calcium hydroxide is observed from about seventh to the $10^{\text {th }}$ day of treatment. [4,5] Recently, there are some newer agents also available for IPT such as, calcium silicate cements (Mineral Trioxide Aggregate, Biodentine ${ }^{\mathrm{TM}}$ and Theracal $\mathrm{LC}^{\mathrm{TM}}$ ), Lasers and Silver Diamine Fluoride (SDF).

Silver diamine fluoride (SDF) $38 \%$ colorless solution been used as effective interim therapy in reducing caries in primary and permanent dentition. It also serves as remineralizing agent. SDF releases calcium fluoride and silver phosphate on contacting carious surface resulting in restoring mineral content and rehardening of the tooth structure. This silver phosphate penetrates partially or completely in compromised dentinal tubules thereby blocking their lumen. [6] It has antimicrobial action that further inactivates cariogenic bacteria. [7] Yamaga et al (1972, 2017), [8,9] described that if SDF is applied in the presence of softened dentin, it will arrest the subsequent progress of dental caries. These properties of SDF might be useful for IPT.

This study therefore aimed at evaluating and comparing clinical and radiographic outcomes of IPT with calcium hydroxide and SDF in primary teeth.

\section{MATERIALS AND METHODS}

This prospective, parallel, randomized controlled study was conducted in the Department of Pediatric and Preventive Dentistry. Study protocol was ethically approved by the Institutional Review Board (TDC/EC/08/2019). Sample size was determined in concordance to results from a previous study (Casagrande $\mathrm{L}$ et al, 2010) [10] through $\mathrm{G}^{*}$ power software (version 3.0.10). A total of 50 primary molars were randomly divided into two groups of 25 each with level of significance (alpha) set at 0.05 and power of the study (1- beta) at $80 \%(0.8)$.

Four to seven years old healthy children with deep occlusal carious lesion on primary molars having clinical symptoms of pain in response to mastication which subsided on withdrawal of stimulus were included in the study. Radiographically, inclusion criteria were carious lesion involving more than two third thickness of dentin approaching the pulp, roots with normal lamina dura and periodontal ligament space. Roots with at least two third of root present were also considered as inclusion criteria for the study.

The exclusion criteria were children with severe gag reflex and children with Frankl behavior rating 1 and 2,. Teeth with proximal carious lesion, presence of pulp exposure, mobility, sinus, abscess, fistula or swelling of periodontal tissues were excluded from the study. Clinical symptoms of irreversible pulpitis like spontaneous pain, nocturnal pain, lingering pain were also excluded. Teeth were excluded if radiographic examination revealed, presence of interradicular or periapical radiolucency, thickening of periodontal ligament space, presence of pathologic root resorption, children having history of allergy to local anesthetic agent.

The procedure was explained in detail to the parents and child in language best understood by them. Written informed consent from the parent and assent of child was obtained before starting the procedure. Sequentially numbered opaque sealed envelopes were used in this study for random allocation of samples to a particular group.

A single experienced operator carried out procedures for both the groups. As the technique of IPT was different in both groups, the operator could not be blinded in this study. 
The procedure commenced with application of $20 \%$ benzocaine gel (Precaine $\mathrm{B}$, Pascal International, USA) at the site of local anesthesia administration for topical anesthesia. Infiltration using $2 \%$ lignocaine with 1:80,000 adrenaline (Lignox 2\% ADR; Warren Pharmaceutical Pvt Ltd, Mumbai, India), was then delivered followed by rubber dam isolation (Hygienic; Coltene/ Whaledent AG, Altstatten, Switzerland). Caries excavation was accomplished in two steps starting with peripheral removal of caries using BR 46 Diamond round point (Mani Inc., Japan) and airotor handpiece (NSK, Japan). This was followed by central excavation using EXC 127/8 sharp spoon excavator (Hu-Friedy Mfg. Co., LLC, Chicago, IL) to judiciously remove soft, necrotic and infected demineralized dentin lying over the pulp tissue with care to avoid a pulp exposure.

For group A, IPT was performed using $38 \%$ SDF solution (FAgamin $\AA$, Tedequim, Córdoba, Argentina). In order to prevent tissue staining and tissue irritation with SDF, a coat of petroleum jelly was applied all over the nearby gingiva, oral mucosa and lips. Applicator tip was wetted with SDF solution and applied over the deepest layer of cavity. The solution was allowed to stay for two minutes after which the excess was removed with cotton pellet. The cavity surface was then gently air dried. Permanent restoration was then done using resin modified glass ionomer cement (GC Gold Label II LC, GC Corporation, India).

For group B, IPT was performed using hard setting calcium hydroxide (Dycal® Radiopaque Calcium Hydroxide, Dentsply Sirona, India). Calcium hydroxide (Dycal) was freshly mixed and applied over deepest layer of cavity using a dycal applicator (GDC marketing, India). The thickness of calcium hydroxide layer was kept approximately at $1.5 \mathrm{~mm}$. [11] The cavity was then restored with resin modified glass ionomer cement.

A postoperative intraoral periapical radiograph (IOPA) was taken at baseline using paralleling technique. Radiographs were standardized by using positioning indicator device (XCP Kit Rinn FPS 3000 xray positioner, Dentsply Sirona, India). The samples were re-examined both clinically and radiographically at baseline, three and six months interval. An investigator blinded to the study performed the clinical and radiographical follow-up examinations.

Clinical success was determined if the tooth revealed absence of spontaneous or night-time pain, sensitivity to pressure or any stimulus, mobility, abscess, sinus, fistula and swelling of periodontal tissue in each follow-up examinations. Radiographic success was determined if the tooth in the IOPA revealed increase in the remaining dentin thickness, absence of interradicular radiolucency, periodontal ligament space thickening and signs of pathological root resorption in each follow-up radiograph.

Any tooth that did not present either of the above clinical or radiographic successful outcomes was recorded as treatment failure and underwent pulpectomy or extracted later.

A blinded analyst carried out data analysis using SPSS version 17.0 software. Clinical and radiographic outcomes of IPT with SDF and calcium hydroxide were evaluated and compared using Fisher's exact test. Level of significance was set at $\mathrm{p}<0.05$.

\section{RESULTS}

The study was conducted to compare the clinical and radiographic outcomes of SDF and calcium hydroxide as an IPT agent on primary molars at six months. The mean age of patients recruited for the study was 5.7 years. The study was started with a total of 25 samples in each group. At follow-up at baseline, no clinical or radiographic failure was reported in both the groups.

At three months follow-up, (Table 1) group A showed one case of clinical failure due to presence of nocturnal pain with the treated tooth. In group B, at three months follow-up two cases of clinical failure were reported. One tooth failed because of 
nocturnal pain and other had developed a sinus.

At six months follow-up, (Table 1) group A showed no treatment failures. In group $\mathrm{B}$, one tooth showed presence of spontaneous pain which was regarded as a treatment failure. Thus, at the end of 12 months, 24 teeth $(96 \%)$ showed success in group A as compared to 22 successfully treated teeth $(88 \%)$ in group B.

No dropouts or loss to follow-up were recorded till six month recall period. Statistical analysis revealed no significant difference in the clinical and radiographic outcomes of both the groups at six months follow up.

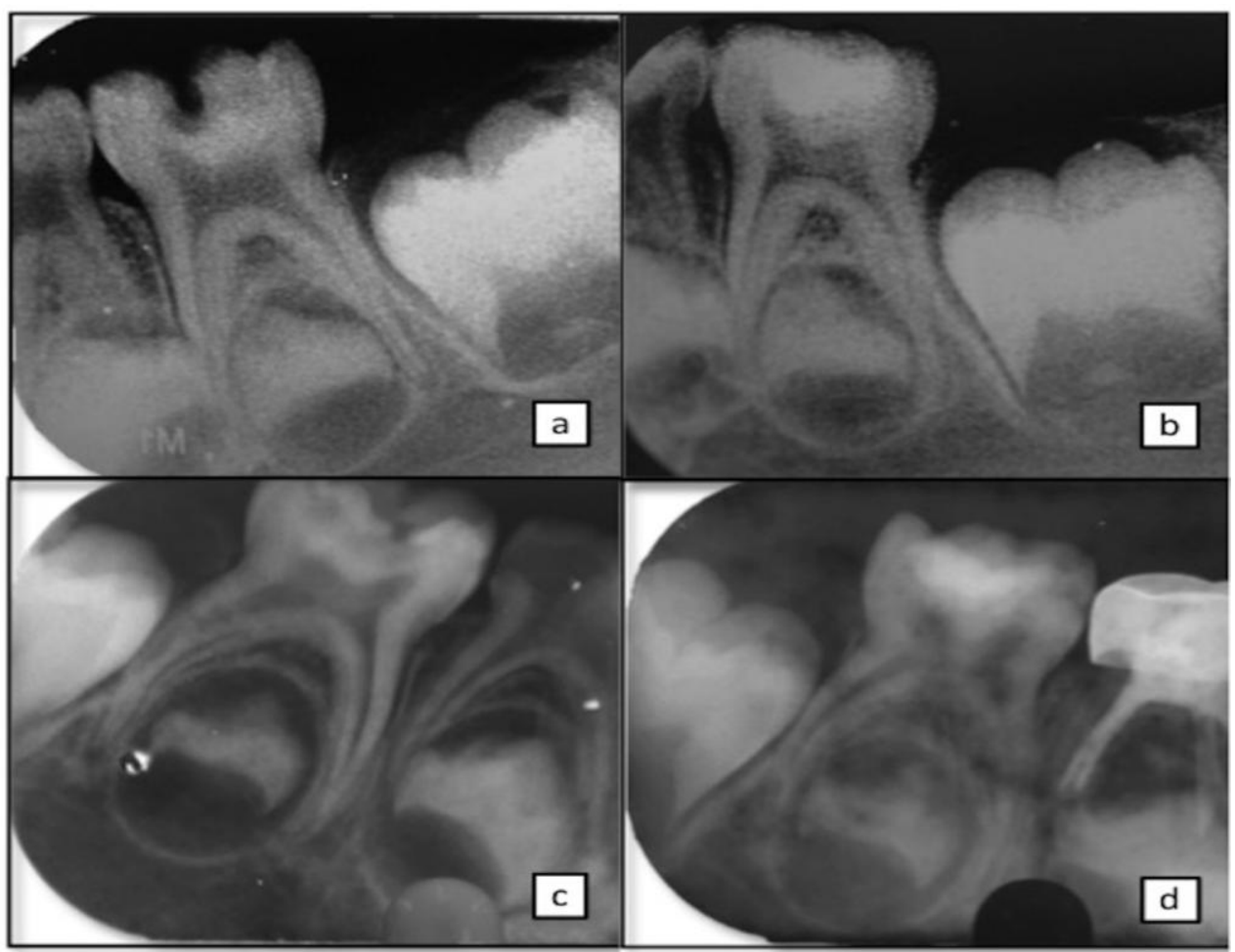

Fig 1: Radiographic examination 1a: preoperative radiograph of group A case, 1b: six months follow up radiograph of group A case, 1c: preoperative radiograph of group B case, 1d: six months follow up radiograph of group B case.

Table1: Results of clinical and radiographic outcomes

\begin{tabular}{|c|c|c|c|c|c|}
\hline Groups & $\begin{array}{c}\text { No. of } \\
\text { teeth }\end{array}$ & $\begin{array}{c}\text { Success 3 month } \\
\text { follow-up }\end{array}$ & $\begin{array}{c}\text { Failure } \\
\text { 3 month follow-up }\end{array}$ & $\begin{array}{c}\text { Success 6 month } \\
\text { follow-up }\end{array}$ & $\begin{array}{c}\text { Failure } \\
\text { 6 month follow-up }\end{array}$ \\
\hline $\mathrm{A}-\mathrm{SDF}$ & 25 & $24(96 \%)$ & $1(4 \%)$ & $24(96 \%)$ & $1(4 \%)$ \\
\hline $\mathrm{B}-\mathrm{Ca}(\mathrm{OH})_{2}$ & 25 & $23(92 \%)$ & $2(8 \%)$ & $22(88 \%)$ & $3(12 \%)$ \\
\hline Total & $\mathbf{5 0}$ & $\mathbf{4 7}(\mathbf{9 4 \%})$ & $\mathbf{3 ( 6 \% )}$ & $\mathbf{4 6}(\mathbf{9 2 \%} \%$ & $\mathbf{4}(8 \%)$ \\
\hline
\end{tabular}

\section{DISCUSSION}

IPT works on the principle of promoting remineralization and arresting the carious process. The disease free collagen fibers act as a base for apatite crystals attachment. The living odontoblastic processes supply calcium phosphate from the vital pulp to aid in physiologic remineralization. [11]

A concern in IPT is the fate of microorganisms present in the deepest layer. Fusayama et al stated that softening of dentin always precedes bacterial invasion and leaves a layer that is soft but not
infected.[12]However, few remaining microorganisms still might persist in deepest layer which usually lose viability in the absence of nutrient source.[4,13]The pulp capping material may also denature the softened carious dentin layer and promote regeneration by laying down reparative or tertiary dentin.[4] Studies have therefore concluded that deepest layer of carious dentin in vital tooth is rendered harmless if sealed with an appropriate pulp capping agent followed by proper coronal sealing. $[4,12,13]$ 
In the present study, the success rates of IPT with calcium hydroxide after six month follow-up period were 88 percent. The findings were similar to the study by George et al (2015), who achieved success rate of $88.3 \%$ at six months.[14]Variable success rates have been observed ranging from $78 \%$ at four years follow up in a study of Casagrande et al to $94.4 \%$ at 12 months follow up by Boddeda et al to $100 \%$ at six months follow up by Chauhan et al.[9,15,16]Factors that are held responsible for the failure of pulp capping procedure with calcium hydroxide include (1) non adherence to dentine, (2) dissolution over time, (3) tunnel defects, (4) necrosis of adjacent pulp tissue, which may lead to compromised vascularity, (5) dislodgement of particles within the body of the pulp. Misdiagnosis of pulpal status is also a factor causing failure of IPT irrespective of the agent being used.[17]

To overcome the failures of the traditional materials, $38 \%$ SDF was evaluated for its efficacy as an IPT agent in this study. SDF has been proved to effectively arrest caries in primary molars by Craig et al and Gotjamanos by reducing demineralization and collagen destruction.[18,19,20]SDF also has antimicrobial effect against cariogenic $S$. mutansor A. naeslundii biofilm on dentin surfaces.[7] These properties might aid in the success of SDF as an IPT agent.

Gupta et al concluded that SDF has greater remineralization potential than that of calcium hydroxide in terms of increased mineral content (fluoride) \& microhardness value when applied to artificially demineralized human dentin.[6] Sinha et al in an in vivo study found that SDF resulted in higher phosphate and fluoride ion levels and lesser calcium ion level as compared to calcium hydroxide in the remaining dentin thickness in permanent molars.[11] Both these studies showed that SDF can be tried as an IPT agent and hence was evaluated in the present study.

IPT with SDF showed a success rate of $96 \%$ in the present study. The main drawback of SDF noticed in this and other studies is discoloration of tooth surface. Other adverse effects of SDF stated in literature include pulpal irritation, tissue irritation.[21]One case in this study showed failure due to presence of irreversible pulpitis symptoms which can be linked to Rossi et al findings, who warned about possible pulpal injury associated with SDF.[21] On contrary, Korwar et al in his ex vivo study found absence of inflammation or necrosis of pulp in cavities treated with SDF.[22]

Hence more clinical trials evaluating the toxicity and biocompatibility of silver compounds are needed to consider safe and widespread use of SDF as an IPT agent in primary molars.

\section{CONCLUSION}

Based on the findings of the present study, the following conclusions can be made:

- IPT with SDF and calcium hydroxide showed similar clinical and radiographic outcomes in primary teeth.

- SDF can be used as an effective alternative to calcium hydroxide for IPT in primary teeth.

\section{Acknowledgement: None}

Conflict of Interest: None

\section{Source of Funding: None}

\section{Ethical Approval: Approved}

\section{REFERENCES}

1. Fusayama T. Two layers of carious dentin: Diagnosis and treatment. Oper Dent. 1979; 4:63-70.

2. Miyauchi H, IW Aku M, Fusayama T. Physiological recalcification of carious dentin. Bnl Tokyo Med Dent Univ. 1978; 25:169-179.

3. American Academy of Pediatric Dentistry. Clinical guidelines on pulp therapy for primary and permanent teeth: Reference manual 2006-07. Pediatr Dent.2006; 28:144-148.

4. Estrela C, Sydney GB, Bammann LL, et al. Mechanism of action of Calcium and 
Hydroxyl ions of Calcium hydroxide on tissues and bacteria. Braz Dent J. 1995; 6:85-90.

5. Ingle JI, Bakland LK. Endodontics. 5thed. London: Elsiver Pub; 2002. pp 864-868.

6. Gupta A, Sinha N, Logani A, et al. An ex vivo study to evaluate the remineralizing and antimicrobial efficacy of silver diamine fluoride and glass ionomer cement type VII for their proposed use as indirect pulp capping materials - Part I. J Conserv Dent. 2011;1 4:113-116.

7. Chu $\mathrm{CH}$, Mei L, Seneviratne $\mathrm{CJ}$, et al. Effects of silver diamine fluoride on dentine carious lesions induced by Streptococcus mutans and Actinomyces naeslundii biofilms. Int J Paediatr Dent. 2012; 22:2-10.

8. Yamaga R, Nishino M, Yoshida S. Diamine silver fluoride and its clinical application. J Osaka Univ Dent Sch.1972; 12:1-20.

9. American Academy of Pediatric Dentistry. Chairside guide: silver diamine fluoride in the management of dental caries lesions. Pediatr Dent. 2017; 39(6):478-479.

10. Casagrande L, Westphalen Bento L, Martini Dalpian D, et al. Indirect pulp treatment in primary teeth: 4-year results. Am J Dent. 2010; 23(1):34-38.

11. Sinha N, Gupta A, Logani A, et al. Remineralizing efficacy of silver diamine fluoride and glass ionomer type VII for their proposed use as indirect pulp capping materials-Part II (A clinical study). J Conserv Dent. 2011; 14:233-236.

12. Fusayama T, Okuse $\mathrm{K}$, Hosoda $\mathrm{H}$. Relationship between hardness, discoloration and microbial invasion in carious dentin. J Dent Res. 1966; 45:103346.

13. Burke FM, Ray NJ, McConnell RJ. Fluoride-Containing Restorative Materials. Int Den J. 2006; 56:33-43.

14. George V, Janardhanan SK, Varma B, et al. Clinical and radiographic evaluation of indirect pulp treatment with MTA and calcium hydroxide in primary teeth (in-vivo study). J Indian SocPedodPrev Dent. 2015; 33(2):104-10.

15. Boddeda KR, Rani CR, Vanga NR, et al. Comparative evaluation of biodentine, $2 \%$ chlorhexidine with RMGIC and calcium hydroxide as indirect pulp capping materials in primary molars: An in vivo study. J Indian SocPedodPrev Dent. 2019; 37(1):606.

16. Chauhan A, Dua P, Saini S, et al. In vivo Outcomes of Indirect Pulp Treatment in Primary Posterior Teeth: 6 Months' Followup. ContempClin Dent 218; 9(Suppl 1):S69S73.

17. Sangwan P, Sangwan A, Duhan J, et al. Tertiary dentinogenesis with calcium hydroxide: A review of proposed mechanisms. IntEndod J. 2013; 46:3-19.

18. Craig GG, Powell KR, Cooper MH. Caries progression in primary molars: 24-month results from a minimal treatment programme. Community Dent Oral Epidemiol. 1981; 9:260-5.

19. Gotjamanos T. Pulp response in primary teeth with deep residual caries treated with silver fluoride and glass ionomer cement ('atraumatic' technique). Aust Dent J 1996; 41:328-34.

20. Mei ML, Li Q, Chun $\mathrm{CH}$, et al. Antibacterial effects of silver diamine fluoride on multi- species cariogenic biofilm on caries. Ann ClinMicrobiolAntimicrob. 2013; 12:1-7.

21. Rossi G, Squassi A, Mandalunis $P$, et al. Effect of silver diamine fluoride (SDF) on the dentin-pulp complex: ex vivo histological analysis on human primary teeth and rat molars. Acta OdontolLatinoam. 2017; 30(1):5-12.

22. Korwar A, Sharma S, Logani A, et al. Pulp response to high fluoride releasing glass ionomer, silver diamine fluoride, and calcium hydroxide used for indirect pulp treatment: An in-vivo comparative study. Contemp Clin Dent 2015; 6:288-92.

How to cite this article: Patil D, Katge F, Jain K et.al. Comparative evaluation of clinical and radiographic outcomes of indirect pulp treatment with silver diamine fluoride versus calcium hydroxide in primary teeth: a randomized controlled trial. International Journal of Science \& Healthcare Research. 2021; 6(2): 150-155. DOI: https://doi.org/ 10.52403/ijshr.20210427 